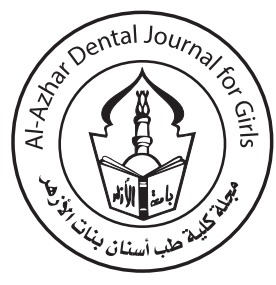

\title{
Remineralizing Ability of Resin Composite Incorporating Calcium Phosphate Nanoparticles and Fluoride Containing Adhesive
}

\author{
Dalia M. Alramady ${ }^{1 *}$, Maha A. Niazy ${ }^{2}$, Asmaa A. Yassen ${ }^{3}$, Mai S. Attia ${ }^{4}$.
}

Codex : 05/22.01

azhardentj@azhar.edu.eg

http://adjg.journals.ekb.eg

DOI: 10.21608/adjg.2021.56032.1328

Restorative Dentistry

(Removable Prosthodontics, Fixed

Prosthodontics, Endodontics, Dental Biomaterials, Operative Dentistry)

\section{KEYWORDS}

Calcium phosphate

nanoparticles, Fluoride,

Mineral density

\begin{abstract}
Purpose: This study was carried out to compare between the remineralizing ability of incorporating calcium phosphate nanoparticles (nCaP) into composite and composite without calcium phosphate nanoparticles, using adhesive with and without fluoride on remineralization of caries affected dentin in-vivo. Materials and Methods: The nanoparticles were incorporated into the flowable i-Flow ${ }^{N}$ composite at a mass fraction of $10 \%$. Forty molars in forty patients having class I caries with ICDAS II score 3 were selected. In each selected molar, caries was removed except from the pulpal floor. Thereafter, molars were divided into two groups $(n=20)$ according to the type composite; resin composite incorporating $\mathrm{nCaP}$ and resin composite without $\mathrm{nCaP}$. Each group was further subdivided into two subgroups $(\mathrm{n}=10)$ according to the adhesive used; resin composite incorporating $\mathrm{nCaP}$ and free fluoride adhesive, resin composite not incorporating $\mathrm{nCaP}$ and free fluoride adhesive. All cavities were finally sealed with nanohybride resin composite (Filtek Z350). Assessment of mineral density was performed at base line $(\mathrm{C})$, after one month $(\mathrm{C} 1)$ and after three months $(\mathrm{C} 3)$ using digitized radiography. Results: The resin composite incorporating nCaP with adhesive induce the highest remineralization potential during the study intervals followed by resin composite incorporating $\mathrm{nCaP}$ free adhesive, then composite free $\mathrm{nCaP}$ with fluoride adhesive and lastly composite not incorporating $\mathrm{nCaP}$ free fluoride adhesive. Conclusion: nanoparticles are capable of remineralizing the caries affected dentin when incorporated into composite resin and fluoride increase the remineralizing ability.
\end{abstract}

* Paper extracted from Doctor Thesis titled "Remineralizing Ability of Resin Composite Incorporating Calcium Phosphate Nanoparticles and Fluoride Containing Adhesive."

1. Doctor in the Ministry of Health, Cairo, Egypt.

2. Professor of Operative Dentistry, Faculty of Dental Medicine for Girls, Al-Azhar University, Cairo, Egypt.

3. Professor of Operative Dentistry, Professor of Conservative Dentistry, Faculty of Dentistry, Cairo University, Cairo, Egypt.

4. Associate professor of Oral Medicine, Periodontology, Diagnosis and Radiology Faculty of Dental Medicine for Girls, AlAzhar University, Cairo, Egypt.

* Corresponding author email: dalia.alramady@yahoo.com 


\section{INTRODUCTION}

The major challenge for the modern approach in restorative dentistry is to induce the remineralization of hypomineralized carious dentine, and therefore, protecting and preserving the vital pulp, so the remineralization of demineralized dentine is the process of restoring minerals through the formation of inorganic mineral-like ${ }^{(1)}$.

Nanotechnology, also known as molecular nanotechnology or molecular engineering, is the development of usable materials and structures in the range of 0.1 to 100 nanometers (nanoscale) by various physical or chemical methods. Recently nanoparticles incorporated into the preventive therapeutic agents and dental restorative materials due to their great potential to reduce biofilm accumulation, decrease the demineralization of the hard dental tissues and enhancement of the remineralization process ${ }^{(2)}$. Calcium phosphate composites have recently been produced with filler particles varying in size from about 1 to $55 \mu \mathrm{m}$. These novel compounds are believed to release high levels of calcium (Ca) and phosphate $\left(\mathrm{PO}_{4}\right)$ ions and able to promote mineral recovery and reduce lesion depth of enamel lesions ${ }^{(3)}$.

Fluoride-containing adhesive releases fluoride when it comes into contact with the wall of the cavity and is followed by its absorption into dentin, fluoride was able to protect the underlying dentin closer to the materials. In this way fluoride from adhesive systems could have some positive effect in the early stages of erosive lesions ${ }^{(4)}$. The goal of this study was therefore to evaluate the remineralizing ability of the resin composite containing calcium phosphate nanoparticles in comparison with resin composite without calcium phosphate nanoparticles applied to affected dentin by using radiograph, this property was examined on using fluoride containing and fluoride free adhesives in- vivo.

\section{MATERIALS AND METHODS}

\section{Preparation of Nanoparticles}

Nanoparticles of amorphous calcium phosphate (nCaP) were chemically prepared in continuous flow-type tube reactor from $50 \mathrm{mM}$ calcium nitrate tetrahydrate $\mathrm{Ca}\left(\mathrm{NO}_{3}\right)_{2}$ and $30 \mathrm{mM}$ diammonium hydrogen phosphate $\left(\mathrm{NH}_{4}\right)_{2} \mathrm{HPO}_{4}$ solutions which fed into $10 \mathrm{~m}$ polytetrafluoroethylene (PTFE) tube under $\mathrm{pH} 9$, at the temperature ranging from 80 to $100^{\circ} \mathrm{C}$. The residence time was approximately 8 $\mathrm{min}$, and the $\mathrm{pH}$ of the reaction solution was regulated at 9 by adding to the phosphate solution ammonium hydroxide. The resulting suspensions were filtered with a membrane filter after the completion of the reaction (Pore size; $0.1 \mathrm{~m}$, ADVANTEC, Japan) and the collected products were rinsed with distilled water before being subjected to vacuum freeze-drying ${ }^{(5)}$.

\section{Preparation of resin composite containing $\mathrm{nCaP}$ :}

$10 \%$ of $\mathrm{nCaP}$ were calculated by mass fraction of composite paste weight, they were put into dark glass bottle which sealed with aluminum foil. Composite paste was added into the bottle containing the calculated powder, and then sonicated together within the glass bottle. The mixture was performed for 10 min using the autoclavable ultrasonic tip of high speed ultrasonicator in a lightproof environment at room temperature. Immediately after the incorporation, the mixture was aspirated again into the original composite syringe, and then the syringe was closed securely again until used.

\section{Characterization of Nanoparticles:}

\section{Transmission Electron Microscope (TEM):}

The scale of the particle morphology was examined by a transmission electron microscope (TEM) operating at $80 \mathrm{kV}$ (model JEM-1230, Jeol, Tokyo, Japan) ${ }^{(6)}$.

\section{Patients' Selection:}

Patients enrolled in this study were chosen from an outpatient clinic in the Operative Department, Faculty of Dental Medicine for Girls, Al-Azhar University. A total of 40 patients aged 20-37 years were chosen for this study. Each patient should have one molar containing occlusal caries with ICDAS 
score 3, no spontaneous pain, no mobility and no percussion tenderness. Radiographically, no internal or external resorption, no periapical or furcation radiolucent and no widening of periodontal ligament space ${ }^{(7)}$, with a total of 40 molars for the study. Patients were told of clinical protocols, experimental reasoning and potential risks. Then were asked to sign a written consent. The approval of the Ethics Committee was received from the Faculty of Dental Medicine for Girls, Al-Azhar University.

\section{Randomization and Blinding:}

The randomization was done using block randomization and the unit was the participant (A sequence no. from 1 to 40 was created and assigned randomly to two blocks with 20 participants in each one to be distributed among the 4 groups using the Microsoft ${ }^{\circledR}$ Excel program). Group A1: with calcium phosphate nanoparticles, or group A2: without calcium phosphate nanoparticles, of twenty participants each according to the type of composite used. Every group was subdivided into 2 subgroups according to the adhesives that would be added to the carious affected dentin; B1: fluoride adhesive, B2: fluoride-free adhesive of ten participants each. Participants were tested immediately $\mathrm{C} 1$ (baseline assessment), C2 (after one month) and after 3 months (C3). A total of 120 readings were collected from the assigned 40 participants.

Primary investigators, participants and outcome assessors were blinded to the allocation. The primary investigator was blinded by storing the restorative materials in identical containers with different numbers. One of the co-authors was responsible for determining the treatment through providing the principle investigator the treatment choice through an opaque sealed envelope.

\section{Cavities preparation:}

In all selected molars, caries were removed using sterile high-speed bur with round bur \# 023 (Komet, Lemgo, Germany) (1 bur/5 cavities) under water coolant. Caries had been removed from all areas of the cavity except that on the pulp floor, where only the excess soft biological material was extracted, then the caries indicator dye (Sable ${ }^{\mathrm{TM}}$ Seek ${ }^{\mathrm{H}}$, Ultradent, USA) was utilized to identify the type of carious dentin at the pulpal floor: either affected or infected.

\section{Application of etching:}

For fluoride free adhesive (Single bond universal, 3M ESPE, Germany), selective etching of enamel margins was performed. For the adhesive containing fluoride $\left(\right.$ ExciTE $\left.^{\mathrm{R}} \mathrm{F}\right)$, total etching was performed. Phosphoric acid gel was applied to the prepared enamel and then flew to the prepared dentin; the manufacturer's instructions were followed precisely. The etchant should be left to react on the enamel for 30 seconds and on the dentin for $15 \mathrm{sec}$ onds, after which the etchant should be removed with a vigorous water spray for at least 5 seconds. Excess moisture can be extracted from the dentin surface with a shiny wet appearance (wet bonding) using a mini sponge.

\section{Application of the Adhesive:}

The adhesives were added to the prepared cavities, after the isolation by rubber dam. The manufacturer's instructions have been followed exactly. Adhesive was applied using a disposable applicator brush and was rubbed for 20 seconds. Solvent dryness and air thinning were achieved by a gentle stream of air over the adhesive for about 5 seconds until it no longer moved and the solvent has evaporated completely, then it was cured for $10 \mathrm{sec}$.

\section{Application of resin composite:}

Calcium phosphate nanoparticles with i-flow composite (i-dental, Karaliauciausstr, 29 Siauliai, Lithuania) were introduced in approximately $1 \pm 0.5 \mathrm{~mm}$ thickness and light cured for 20 seconds using a 470 wavelength (Elipar 3M, ESPE) LED light-curing device with a light intensity of $1000 \mathrm{~mW} /$ $\mathrm{cm} 2$. Filtek Z250 XT, (3M ESPE, USA) nanohybrid 
resin composite was applied to the cavity using teflon tipped unit. Oblique increments $(2 \mathrm{~mm} / \mathrm{each})$ of resin composite were inserted and light-cured $(20 \mathrm{~s})^{(8)}$ to complete the cavity and cured as indicated above, then finished with a high-speed handpiece with a water coolant using a flame stone finish (No. 833C FG, sizes \#014 and \#016, Meisinger GmbH, Germany) and fine tapered stone with a round end (805C FG, size \#012, Meisinger GmbH, Germany). Polishing was done using single-step polishing tips and/or cups (Dimanto ${ }^{\circledR}$, Voco Gmbh, Germany) worked at low-speed contra-angle handpiece (NACEC, NSK, Japan) with a speed not exceeding 20,000 rpm with water coolant and minimal pressure. Occlusion was tested using articulating paper (Blue Red Combo 0.0028"/71, Crosstex ${ }^{\circledR}$ International, USA) and high spots were removed.

\section{Radiographic assessment:}

Patients were examined radiographically, using an intra-oral optical radiograph (X MIND, France) with exposure parameters of 70 kilovoltage (Kvp) and 0.63 milliampere $(\mathrm{mA})$ using an image plate (size 2) (3x4) (Durr Dental, Germany). These images were then moved to the digital scanner. Barrier envelopes were used to wrap the image plate when imaging to avoid cross-infection (Image plate safety bag, No. SDT-XR57, smile dental, NiHEN). A posterior parallel XCP package (Dentsply International Inc, USA was used for standardization. After irradiation using the posterior parallel kit, the plate was removed from the barrier envelope, scanned by Durr Vista can Combi Plus Dental CR (No. 2150- 04, Durr Dental, Germany), and the image was stored on the device. Radiographic procedures were conducted at baseline, 1 and 3 months intervals.

\section{Assessment of remineralization:}

The DBSW in software was used to perform measurements (fig1). In each sample, a line was drawn along the CEJ to serve as a reference line, while another parallel line was drawn at the bottom of the cavity. The distance between the two lines was standardized (for each sample) by measuring the length of the vertical line connecting them. The length of the line centrally located at the bottom of the cavity (in pixels) was calculated directly by means of the above mentioned software. Such length has also been set for each sample during the assessment intervals. Three points on this line beginning, middle and end) were calculated. The intensity at each of these points was calculated by taking the corresponding three readings from the DBSW software and recording them to measure the average intensity. These procedures were performed at baseline $(\mathrm{C} 1)$ and repeated at one month $(\mathrm{C} 2)$ and three months $(\mathrm{C} 3)$ intervals. The mean mineral density value was determined from the equation (average density $=$ average intensity/profile); (the profile was determined directly in the utilized software).

Statistical research was then carried out using a commercially accessible software programme using IBM SPSS software package version 20.0. Since the image density data is parametric, the significance of the disparity between different groups was assessed using a one-way variance analysis (ANOVA) test followed by a Tukey post-hoc test when ANOVA suggested a significant difference. The significance level was set at $\mathrm{P}<0.05$.

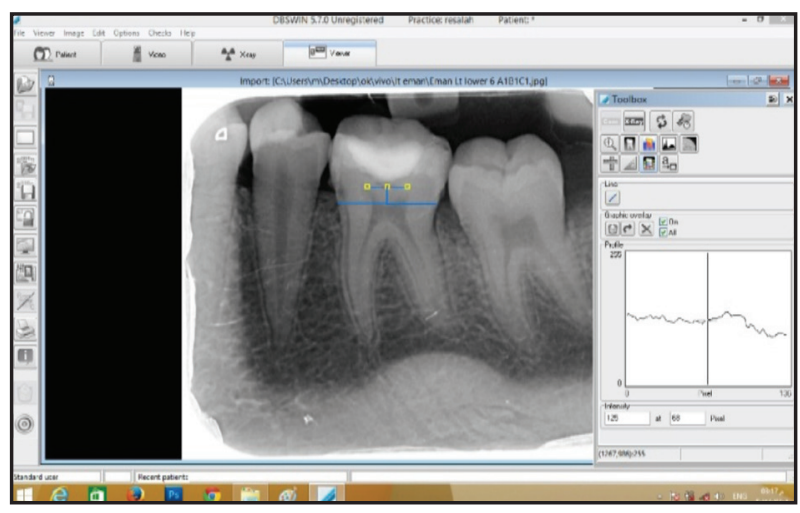

Figure (1) An image showing the way of measurement using DBSW in Programme. 


\section{RESULTS}

Mean, standard deviation and level of significance of the change on mineral density in pixels with different composite and adhesive type are shown in table (1) and fig 2. It was noticed that, there was no statistically significant difference between the tested groups at base line readings at $\mathrm{P}(0.571)$.

After one month, it was revealed that composite incorporating calcium phosphate nanoparticle (nCaP) accompanied with fluoride containing adhesive recorded the highest mineral density mean value $(0.80 \pm 0.13)$ with a non-statistical difference between it and that of composite incorporating calcium phosphate nanoparticle ( $\mathrm{nCaP}$ ) accompanied with fluoride free adhesive $(0.76 \pm 0.14)$, and followed by composite without calcium phosphate nanoparticle (nCaP) accompanied with fluoride containing adhesive $(0.67 \pm 0.17)$. On the other hand, composite without calcium phosphate nanoparticle
(nCaP) accompanied with fluoride free adhesive recorded the statistically lowest mineral density $(0.63 \pm 0.13)$. Difference between the study groups after one month was statistically significant at $\mathrm{P}(0.041)$.

Furthermore, after three months, it was revealed that composite incorporating calcium phosphate nanoparticle ( $\mathrm{nCaP}$ ) accompanied with fluoride containing adhesive recorded the highest mineral density mean value $(1.14 \pm 0.50)$ with no statistical difference with that of composite incorporating calcium phosphate nanoparticle ( $\mathrm{nCaP}$ ) accompanied with fluoride free adhesive $(0.95 \pm 0.35)$. On the other hand, composite without calcium phosphate nanoparticle (nCaP) accompanied with fluoride containing or fluoride free adhesives recorded statistically lower mineral density values $(0.70 \pm 0.16)$ and $(0.63 \pm 0.13)$ respectively. Difference between the study groups after three months was statistically significant at $\mathrm{P}(0.004)$.

Table (1) Mean standard deviation and level of significance of the comparison of mineral density results between study groups.

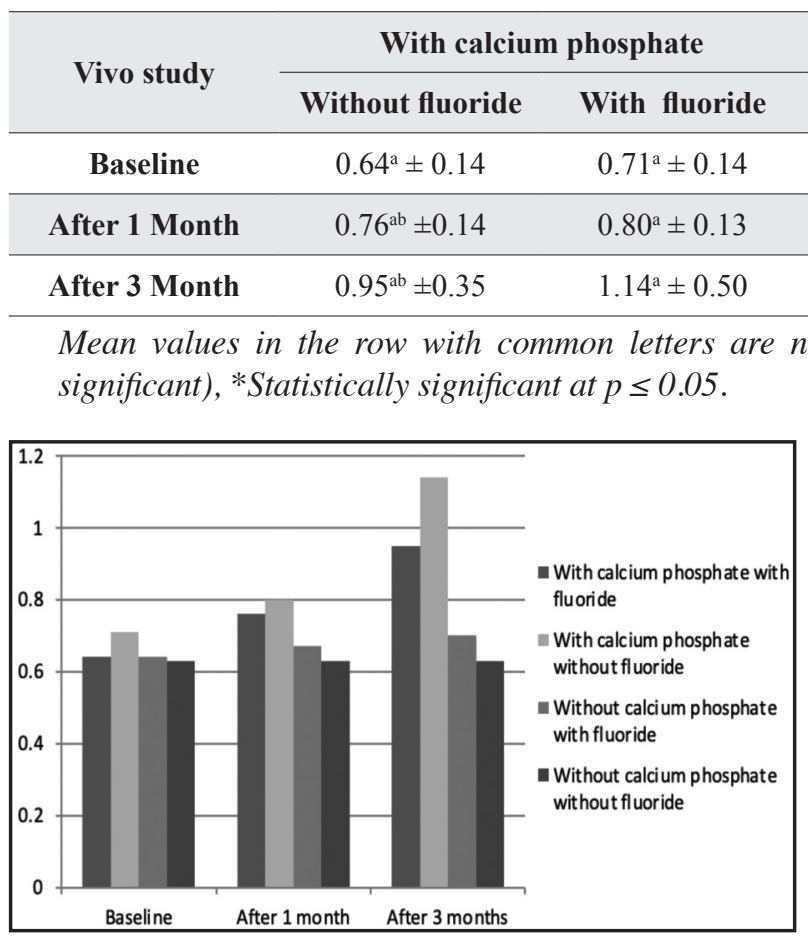

Figure (2) Bar chart of mineral density mean values between study groups.

\section{DISCUSSION}

Demineralization is a reversible mechanism that favors remineralization if damaged tissues are exposed to an oral environment. For example, enamel cavities created by demineralization processes are naturally remineralized by outgrowth of residual crystals acting as nucleation sites, while saliva provides a supersaturated $\mathrm{Ca}_{2}$ and $\mathrm{PO}_{4}{ }^{3-}$ ion environment compared to hydroxyapatite $(\mathrm{HA})^{(9,10)}$. However, the remineralization of enamel with saliva is rarely fully accomplished, particularly when 
the extent and duration of the demineralization/ remineralization phases are imbalanced ${ }^{(9,10)}$.

Therefore, the remineralization process require using an external source of $\mathrm{Ca}^{2+}$ and $\mathrm{PO}_{4}^{3-}$ ions to maximize HA supersaturation so as to effectively avoid demineralization and improve remineralization ${ }^{(9)}$. Its ability also be accomplished by using different types of biomimetic calcium phosphate $(\mathrm{CaP})$ mimicking the composition and texture of bone and dental mineral phases (so-called biomimetic), biomimetic analogues must be necessary to achieve functional mineralization and to recover the dynamic mechanical properties of teeth and seems to be a promising approach for both enamel and dentin remineralization such as: HA, fluoro-hydroxyapatite (FHA) and amorphous calcium phosphate (ACP) ${ }^{(11,12)}$. These agents may be added to the restorative material or directly applied to the tooth surface, or use in eradication of microbial biofilms from the root canal system ${ }^{(10)}$.

$\mathrm{CaP}$ nanostructured are more effective compared to their macro-sized counterparts due to their biomimetic, higher surface area and reactivity, as well as improved ability to adhere and penetrate the enamel and dental lesions. Moreover, nanomaterials can interfere with the bacteria metabolism, inhibit the formation of biofilm, reduce demineralization, and promote remineralization, which is expected to be an effective strategy for caries management ${ }^{(13)}$. The goal of this study was therefore to evaluate the impact of the incorporation of amorphous calcium phosphate nanoparticles (nCaP) into the composite with and without fluoride adhesive to the affected dentin.

Clinically, coronal dentin lesion can be separated into two structurally distinct regions. The outer, shallow zone that has endured the ravages of a carious attack or the longest period of time and eventually consists of irreversibly acid demineralized dentin. Collagen in this zone has been subjected to excessive proteolytic degradation, which prevents any reconstruction of the molecular crosslinks. The inner, deeper zone of the lesion is made up of dentin, which has been reversibly assaulted by a carious mechanism and can therefore be remedied under favorable conditions. It is thought that this zone should be maintained on the cavity floor in order to allow remineralization after an adequate restoration has been applied ${ }^{(14)}$. Digital radiograph can be used to estimate the degree of mineralization of the hard tooth structure, assessment of the remineralizing ability of the dental materials, and also was used to measure the radiodensity (DBSW software) ${ }^{(15)}$.

Results of the current study showed that resin composite containing calcium phosphate nanoparticles (nACP) and fluoride adhesive showed the highest mean percent change in mineral density from baseline to 3 months. This means that it was the highest group capable of inducing remineralization to the remaining caries affected dentin, followed by resin composites containing calcium phosphate nanoparticles free fluoride adhesive, then resin composites not incorporating nanoparticles but containing adhesive fluoride and lastly resin composites free of both nanoparticles and adhesive fluoride. This may be due to the high release of $\mathrm{Ca}, \mathrm{PO}_{4}$ and $\mathrm{F}$ ions known for their remineralizing capabilities. The release of $\mathrm{Ca}, \mathrm{PO}_{4}$ and $\mathrm{F}$ ion from composite resins and adhesives can be highly beneficial and may serve as seed crystals to promote remineralization in the hybrid layer and at the tooth-restoration margins. The higher capacity of ACP nanoparticles to induce amorphous remineralization is characterized by a higher rate of dissolution and higher ion release.

These findings were consistent with the results of another study which indicated that the $(\mathrm{nCaP})$ composite had acid neutralization and the $\mathrm{Ca}$ and $\mathrm{P}$ ion release capability ${ }^{(16)}$. Poly(amidoamine) PAMAM) or amorphous calcium phosphate (nCaP) nanoparticles, each of which alone achieved remineralization and increased demineralized dentin hardness, PAMAM/nCaP nanocomposite achieved the greatest mineral regeneration on demineralized dentin, and the greatest improvement in hardness was observed in demineralized dentins approaching 
normal dentin hardness. In addition, it was found that novel nACP-based nanocomposites have been shown to achieve dentin lesion remineralization ${ }^{(17)}$.

Poly (amidoamine) (PAMAM) and rechargeable composite incorporating nanoparticles of calcium phosphate (NACP) have also been reported to achieve dentin remineralization completely and restore the hardness similar to healthy dentin, so that PAMAM plus recharged NACP remineralized predemineralized dentin completely, with prolonged fluid challenge like to the oral environment ${ }^{(18)}$. Moreover, it was suggested that dentin desensitizer containing 3000ppm fluoride and methacrylate-cop-styrene sulfonic acid (MS polymer) has the same anti-demineralization effect on root dentin lesion as does a fluoride paste containing $9000 \mathrm{ppm} \mathrm{F}^{(19)}$. Furthermore, it was reported that fluoride dentifrice was capable to hamper further demineralization of sound dentin as well as lowly and highly demineralized dentin artificial caries ${ }^{(20)}$.

The findings, however, came with disagreement with others who claimed that fluoride has also been found to have the highest remineralization trend for both calcium and phosphate, and has been shown to have a remineralizing effect compared to calcium triphosphate and laser IR810 ${ }^{(21)}$. Fluoride released from fluoride-containing dental adhesives did not affect the resistance to demineralization of human tooth structure. Moreover, the released fluoride may adversely influence the integrity of resin/dentine interface and, hence, the bond strength and bond durability of adhesive system to dentine or enamel structure ${ }^{(22)}$.

\section{CONCLUSION}

Nanoparticles are capable of remineralizing the caries affected dentin when incorporated into composite resin. Fluoride adhesive plays an important role in augmentation of the remineralization process. This could be a promising approach for the prevention of secondary caries at the cavity margins. Moreover, nanoparticles still have many aspects and useful applications in the field of dentistry.

\section{CONFLICT OF INTEREST}

None declared.

\section{FUNDING}

No funding was received for this study.

\section{REFERENCES}

1. Cao CY, Mei ML, Li QL, Lo ECM, Chu CH. Methods for biomimetic remineralization of human dentin: A systematic review. Int J Mol Sci. 2015; 16: 4615-27.

2. Khan I, Saeed K, Khan I. Nanoparticles: Properties, applications and toxicities. Arab J Chem. 2019; 12:908-31.

3. Alania Y, Natale LC, Nesadal D, Vilela H, Magalhães AC, Braga RR. In vitro remineralization of artificial enamel caries with resin composite containing calcium phosphate particles. J Biomed Mater Res Part B. 2019; 107:1542-50.

4. Guedes APA, Mode MD, Suzuki TYU, Godas AGD, Sundfeld RH, Birso ALF, et al. Effect of fluoride-releasing adhesive systems on the mechanical properties of eroded dentin. Braz Dent J.2016; 27:135-9.

5. Fujii E, Kawabata KY, Hirosaki YS, Hayakawa S, Oska A. Fabrication of calcium phosphate nanoparticles in a continuous flow tube reactor. J Cera Soc Jap. 2015; 123:101-5.

6. Mostafa AA, Zaazou MH, Chow LC, Mahmoud AA, Zaki DY, Basha M. Injectable nanoamorphous calcium phosphate based in situ gel systems for the treatment of periapical lesions. Biomed Mater. 2015; 10:1-14.

7. Niazy MA, El- Marsafy SMA and Nour El- Deen MM. Clinical and radiographic evaluation of two tissue engineering materials (Bio Gen and Bioaggregate) and direct pulp capping agent in human tooth. J Cairo D.:27;2011 26 17-.

8. Marques IP, de Oliveira FB, Souza JG, Ferreira RC, Magalhães CS, França FM, et al. Influence of surface treatment on the performance of silorane-based composite resin in class I restorations: a randomized clinical trial. Clin Oral Invest. 2018; 16:1-8.

9. Neel EAA, Aljabo A, Strange A, Ibrahim S, Coathup M, Young AM, et al. Demineralization-remineralization dynamics in teeth and bone. Intern J Nanomed. 2016; 11:4743-63.

10. Raura N, Garg A, Arora A, Roma M. Nanoparticle technology and its implications in endodontics: a review. Biomater Res. 2020; 24: 1-8. 
11. Enax, J. and Epple, M. Synthetic hydroxyapatite as a biomimetic oral care agent. Oral Health Prevente Dent. 2018; 16: 7-19.

12. Basheer, N., Madhubala, M. M., \& Mahalaxmi, S. Future perspectives of biomimetics in restorative dentistry. $\mathrm{J}$ Pharml Res Inter.2020; 32: 19-28.

13. Chen H, Gu L, Liao B, Zhou X, Cheng L, Ren B. Advances of anti-caries nanomaterials. Molocules.2020; 25: 1-16.

14. Cheng L, Zhang K, Weir MD, Melo MA, Zhou X, Xu HH Nanotechnology strategies for antibacterial and remineralizing composites and adhesives to tackle dental caries. Nanomedicine. 2015; 10:627-41.

15. Elasser DM, Niazy MA, Elsharkawy DAE, Mansour MS The remineralizing potential of nano bioactive glass versus nanohydroxyapatite on dentine as affected by $\mathrm{PH}$ cycling. A D J. 2018; 5:327-34.

16. Liang K, Weir MD, Xie X, Wang L, Reynolds MA, Li J. Dentin remineralization in acid challenge environment via PAMAM and calcium phosphate composite. Dent Mater. 2016; 32: 1429-40.

17. Weir MD, Ruan J, Zhang N, Chow LC, Zhang K, Chang $\mathrm{X}$. Effect of calcium phosphate nanocomposite on in vitro remineralization of human dentin lesions. Dent Mater. 2017; 33: 1033-44.
18. Liang, K, Xiao S, Wu J, Li J, Weir MD, Cheng L. Longterm dentin remineralization by poly (amido amine) and rechargeable calcium phosphate nanocomposite after fluid challenges. Dent Mater. 2018; 34: 607-18.

19. Kawamura K, Kunimatsu Y, Nakano T, Hasegawa $H$, Arakawa H, Mukai Y. Anti-demineralization effect of desensitizer containing copolymer and sodium fluoride on root dentin - a transverse microradiographic study. Acta Biomater Odonto Scand. 2019; 5: 38-43.

20. Wierichs RJ, Musiol J, Erdwey D, Esteves-Oliveira M, Apel C, Meyer-Lueckel H. Re- and demineralization characteristics of dentin depending on fluoride application and baseline characteristics in situ. J Dent. 2020; 94:1-8.

21. Carrio EL, Rojas NL, Luckie RAM, Bermeo NLR, Fabila MMG, Santillance RR. The effect of remineralization via fluoride versus Low-Level Laser IR810 and fluoride agents on the mineralization and microhardness of bovine dental enamel. Appl Sci. 2018; 8: 1-8.

22. Pirmoradian M, Esmailzadeh S, Davaie S, Albakhakh BAJ, Sanaee B, Asgari E, et al. Resistance to demineralization of adjacent enamel and dentine, fluoride release and dentine bond strength of fluoride-containing self-etch adhesive systems. J Clin Exp Dent. 2020; 12:381-90. 